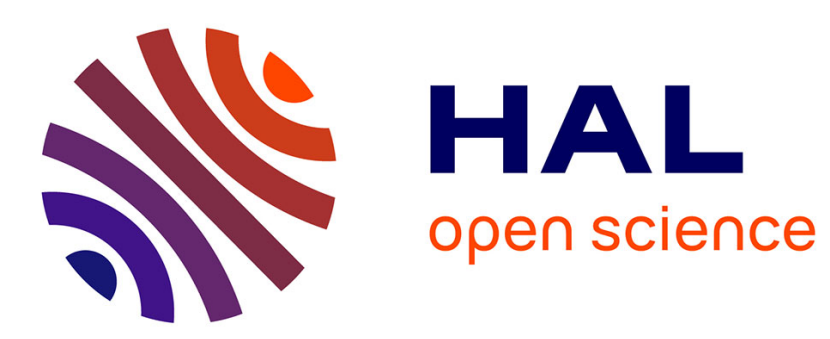

\title{
Modeling and Swimming Property Characterizations of Scaled-Up Helical Microswimmers.
}

Tiantian Xu, Gilgueng Hwang, Nicolas Andreff, Stéphane Régnier

\section{To cite this version:}

Tiantian Xu, Gilgueng Hwang, Nicolas Andreff, Stéphane Régnier. Modeling and Swimming Property Characterizations of Scaled-Up Helical Microswimmers.. IEEE/ASME Transactions on Mechatronics, 2014, 19 (3), pp.1069-1079. hal-01324577

\section{HAL Id: hal-01324577 \\ https://hal.science/hal-01324577}

Submitted on 9 Jun 2016

HAL is a multi-disciplinary open access archive for the deposit and dissemination of scientific research documents, whether they are published or not. The documents may come from teaching and research institutions in France or abroad, or from public or private research centers.
L'archive ouverte pluridisciplinaire HAL, est destinée au dépôt et à la diffusion de documents scientifiques de niveau recherche, publiés ou non, émanant des établissements d'enseignement et de recherche français ou étrangers, des laboratoires publics ou privés. 


\title{
Modeling and Swimming Property Characterizations of Scaled-up Helical Microswimmers
}

\author{
Tiantian $\mathrm{Xu}^{1}$, Gilgueng Hwang ${ }^{2}$, Nicolas Andreff ${ }^{3}$ and Stéphane Régnier ${ }^{1}$
}

\begin{abstract}
Micro and nanorobots capable of controlled propulsion at low Reynolds number are foreseen to change many aspects of medicine by enabling targeted diagnosis and therapy, and minimally invasive surgery. Several kinds of helical swimmers with different heads actuated by a rotating magnetic field have been proposed in prior works. Beyond these proofs of concepts, this paper aims to obtain an optimized design of helical swimmers adapted to low Reynolds numbers. For this, we designed an experimental setup and scaled-up helical nanobelt swimmers with different head and tail coatings to compare their rotational propulsion characteristics. We found in this paper that the head shape of a helical swimmer does not influence the shape of the rotational propulsion characteristics curve, but it influences the cut-off frequency values. The rotational propulsion characteristics of helical swimmers with a magnetic head or a magnetic tail are different. The helical swimmers with uniformly coated magnetic tails do not show a cut-off frequency whereas the ones with a magnetic head exhibit a saturation of frequency.
\end{abstract}

\section{INTRODUCTION}

Micro and nanorobots will have a great impact in medicine. They can be used to targetedly deliver chemical and biological substances, to remove material by mechanical means, to act as simple controllable static structures or to transmit biological data from a specific hard to reach location [1].

The medical context often implies that the micro and nanorobots have to swim in fluid. However, at the micro and nano scale, the fluid become extremely viscous and the Reynolds number of the micro and nanorobots dramatically lower. Purcell described two swimming techniques which are suitable for swimming at low Reynolds numbers [2], [3] : corkscrew type rotating propulsion and oscillation of flagella. Micro and nanoscale helical swimmers are inspired by E. coli bacteria. E.coli bacteria consist of a rod-shaped head and a bundle of passive flagella. Flagella are driven by a rotary motor into a helical shape to generate a corkscrew-like motion [4].

Since more than ten years ago, researchers have developed several different micro helical swimmers, specially with different head shapes. The first helical

\footnotetext{
${ }^{1} \mathrm{~T} . \mathrm{XU}$ and S.Régnier are with ISIR - Institut des Systèmes Intelligents et Robotique, UPMC, Paris, France. tiantian.xulisir.upmc.fr, stephane.regnier@upmc.fr

${ }^{2} \mathrm{G}$. Hwang is with Laboratoire de Photonique et de Nanostructures, Marcoussis, France. gi l gueng. hwangelpn.cnrs.fr

${ }^{3} \mathrm{~N}$. Andreff is with FEMTO-ST Institute, UFC, Besancon, France. nicolas.andreffefemto-st.fr
}

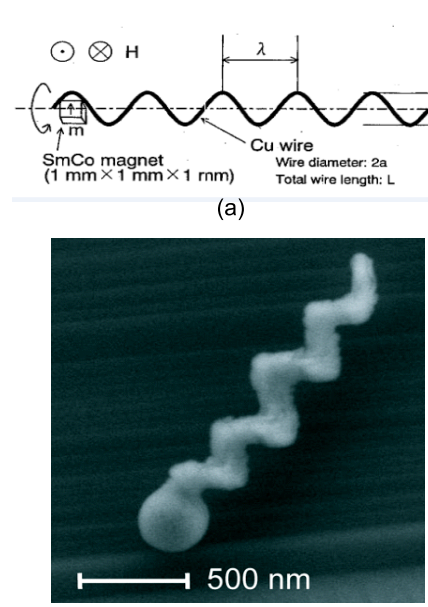

(c)

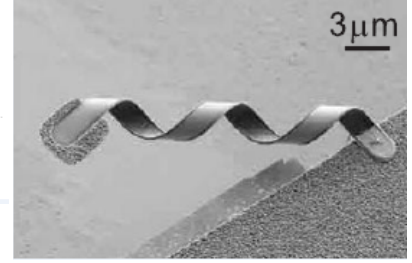

(b)

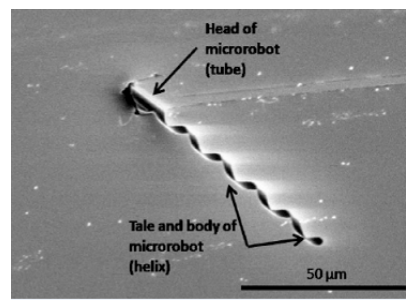

(d)

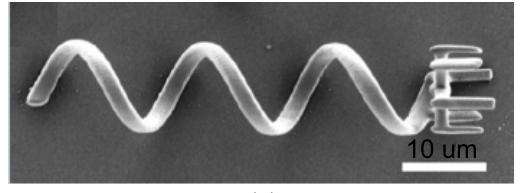

(e)

Fig. 1: (a) The first helical type swimmer with a cubic magnetic head [5]. (b) Artificial Bacterial Flagella with a thin-square-plate soft magnetic head [6], [7]. (c) A smaller helical swimmer with a spherical head [8]. (d) Helical Nanobelt with a cylindrical tube head [9], [10]. (e) A polymer helical swimmer with a "claw" shaped holder head [11].

type swimming machine was proposed by Honda et al. in 1996 [5], as shown in Fig. 1a. This swimming machine was as large as a few millimetres, with a strong permanent magnetic cubic head and a helical tail made of copper filament. This device could be driven wirelessly by an external rotating magnetic field. The swimming performance tests were in low Reynold number conditions. They predicted that this helical type swimming machine with a length of $11.5 \mathrm{~mm}$ could still be scaled down to micrometer-sized machine [12]. Thanks to the recent development in micro and nanotechnology, researchers of ETH Zurich fabricated microscale helical swimmers in 2007, called "Artificial Bacterial Flagella" (ABF), by using a self-scrolling fabrication technique [13], [6], [14], [7]. The ABF consisted of a helical tail made by GaAs/InGaAs and a thinsquare-plate $(4.5 \mu \mathrm{m} \times 4.5 \mu \mathrm{m} \times 200 \mathrm{~nm})$ soft magnetic 
head on one end, as shown in Fig. 1b. The total length of the $\mathrm{ABF}$ was approximately $50 \mu \mathrm{m}$ and its thickness was approximately $30 \mathrm{~nm}$. A group at Harvard presented in 2009 even smaller helical swimmers with a diameter of $200 \mathrm{~nm}$ and a length of $2 \mu \mathrm{m}$ [8]. The swimmers were made of glass $\left(\mathrm{SiO}_{2}\right)$. The swimmers have spherical heads. A permanent magnetic film was evaporated onto one side of the swimmers, as shown in Fig. 1c. Our group at ISIR showed that electro-osmosis propulsion was more efficient than magnetically actuated propulsion [9], [10]. The propulsion achieved a speed of 24 body lengths per second. The micro helical swimmers we used, called "Helical NanoBelt" (HNB), had a cylindrical head and a helical tail. The total length was about $70 \mu \mathrm{m}$. The entire surface was coated by a $10 \mathrm{~nm}$ thick Nickel layer. This surface-coated HNBs, as shown in Fig. 1d, has a similar geometry to ABF, but their entire surface can be functional to propulsion in comparison to the ABF's soft magnetic head as the only functional part and the tail as a passive part. More recently, the ETH group presented a helical swimmer with a "claw" shaped holder attached to a polymer helical tail, as shown in Fig. 1e [11]. This holder was used to transport micro objects.

The control of the helical swimmers' movement has not been clearly realized mainly due to the limited observation tools to characterize the propulsion characteristics and to identify the propulsion matrix at the microscale. This prevents their microrobotic applications which usually need to implement the closed loop control. This paper first proposes to assemble Scaledup Helical Nanobelts (SHNs) without heads and to develop their control testbed to identify their propulsion matrices and to observe their rotational propulsion characteristics. This system revealed the propulsion matrices of two different SHNs with one or four pitch magnetic surfaces. The required forces and torques to drive the SHNs were simulated based on the propulsion matrices. From a methodologic point of view, this paper therefore introduces a novel investigation approach which relies on adimensionality principles.

So far, several different heads for helical swimmers have appeared. However their influence on propulsion characteristics has not been shown yet. Moreover, the difference between the propulsion characteristics of helical swimmers with a magnetic head or with a magnetic tail has not been clarified. Therefore, this paper proposes also to assemble SHNs with different head shapes. Thereby, it is possible to compare their rotational propulsion characteristics, and to find the head shape influence. At last, we propose to compare the rotational propulsion characteristics between the helical swimmers with magnetic heads and those with magnetic tails. Two methods were used to make a magnetic tail. One method consists in covering the helical tail with small magnets, the other one in uniformly coating the helical tail with ferromagnetic material.
Later the rotational propulsion characteristics between the two kinds of magnetic tails were compared.

\section{EXPERIMENTAL SETUP}

\section{A. Scaled-up helical swimmers with different heads}

The swimming behaviour of microscale helical swimmers has not been clearly defined mainly due to the limited observation tools. Moreover, the micro fabrication process for self-rolling HNBs usually takes long. Making different head shapes and different geometries is a challenge for micro-fabrication. That is why Scaledup Helical Nanobelts (SHNs) are designed. Two scales of SHNs were made : SHN10s and SHN2s.

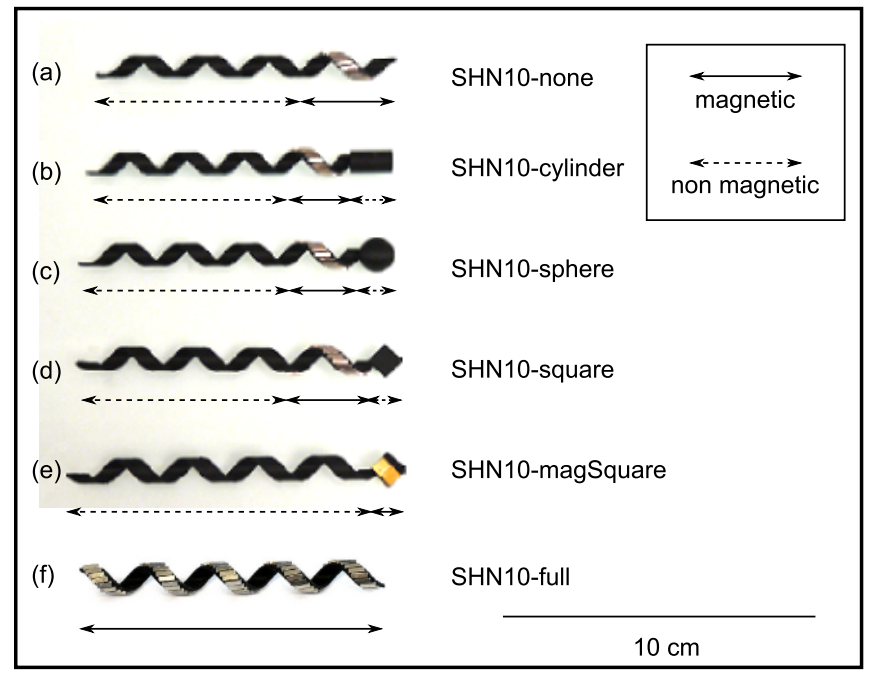

Fig. 2: The Scaled-up helical nanobelts of $10 \mathrm{~cm}$ length (SHN10s) with different heads : (a) SHN10-none : one pitch magnetic with no head. (b) SHN10-cylinder : with a cylindrical head. (c) SHN10-sphere : with a spherical head. (d) SHN10-square : with a square head. (e) SHN10-magSquare : with a magnetic square head. (f) SHN10-full : full pitch magnetic with no head.

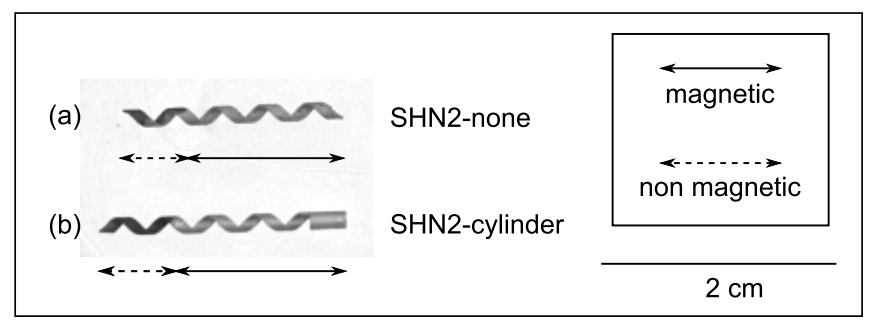

Fig. 3: The Scaled-up helical nanobelts of $2 \mathrm{~cm}$ length (SHN2s) with different heads : (a) SHN2-none : with no head. (b) SHN2-cylinder : with a cylindrical head.

1) SHN10s: SHN10s' basic structures are made of ABSPlus P400 by a rapid prototyping machine (Stratasys Dimension SST 768). The length of SHN10s are about $10 \mathrm{~cm}$. The geometry is scaled up proportionally except for the thickness, because the minimal thickness 
of the fabrication is limited by prototyping machine's capacity. The following two categories of SHN10 were made. The category named SHN10-magTail includes SHN10s with their first pitches (i.e. a 1-full-turn portion of the helix) covered with $\mathrm{NdFeB}$ permanent magnets $(5 \mathrm{~mm} \times 1.5 \mathrm{~mm} \times 1 \mathrm{~mm})$, and a SHN10 with its entire surface covered with $\mathrm{NdFeB}$ permanent magnets. The magnets were stuck on the surface by superglue. In this category, the SHN10s with one pitch magnetic have different head shapes : without a head, with a cylindrical head, spherical head and square head, as shown in Fig. 2. The heads are not magnetic. They are named SHN10-none, SHN10-cylinder, SHN10-sphere and SHN10-square. The SHN10 with its entire surface magnetic and no head is named SHN10-full. The other category, named SHN10-magHead, includes one SHN10 with a square head covered by NdFeB magnets $(5 \mathrm{~mm} \times 4 \mathrm{~mm} \times 1 \mathrm{~mm})$. The whole tail of SHN10magSquare is non-magnetic.

2) SHN2s: SHN2s' basic structures are made of titanium. They were made by i.materialise (3D printing company). They are coated by ferromagnetic material : nickel. The length of SHN2s is about $2 \mathrm{~cm}$. SHN2s were designed smaller in order to make the coating process easier. For the thick layer coating, electroplating was used. Titanium oxides very easily. The titanium was thus deoxidized by hydrochloric acid right before the electroplating. The titanium models were placed onto the negative electrodes and the positive electrode was placed near the models. These were immersed in a nickel bath with the application of electric potential between the two electrodes. The deposition rate was calibrated onto the dummy silicon surface coated with titanium layer. The end of the helical tail is used to make an electric contact during the electrolytic deposition process, thus the last pitch of SHN2 is not coated. Table I summarizes the specifications of the two scales of SHNs.

TABLE I: Specifications of the HNB and the SHNs [9], [15].

\begin{tabular}{l|c|c|c} 
Parameters & HNB & SHN10 & SHN2 \\
\hline Diameter & $2.1 \mu \mathrm{m}$ & $6 \mathrm{~mm}$ & $1.2 \mathrm{~mm}$ \\
Thickness & $27.2 \mathrm{~nm}$ & $1 \mathrm{~mm}$ & $0.2 \mathrm{~mm}$ \\
Pitch & $14 \mu \mathrm{m}$ & $20 \mathrm{~mm}$ & $4 \mathrm{~mm}$ \\
Width & $2.5 \mu \mathrm{m}$ & $7.2 \mathrm{~mm}$ & $1.44 \mathrm{~mm}$ \\
Length & $62 \mu \mathrm{m}$ & $97.2 \mathrm{~mm}$ & $17.8 \mathrm{~mm}$ \\
\hline Nb of turns & \multicolumn{3}{|c}{4.5} \\
\hline Weight & - & (a) $2.08 \mathrm{~g}$ & (a) $0.04 \mathrm{~g}$ \\
& & (b) $1.75 \mathrm{~g}$ & (b) $0.05 \mathrm{~g}$ \\
& & (c) $2.63 \mathrm{~g}$ & (d) $1.85 \mathrm{~g}$ \\
& & (e) $1.33 \mathrm{~g}$ & (f) $4.98 \mathrm{~g}$ \\
\hline Magnetic material & Nickel & NdFeB & Nickel \\
Magnetic layer & $30 \mathrm{~nm}$ & $1 \mathrm{~mm}$ & $20-50 \mu \mathrm{m}$
\end{tabular}

\section{B. Swimming at low Reynolds number}

The Reynolds number is a dimensionless quantity, which is defined as the ratio of the inertial forces $\left(f_{i n}\right)$ to the viscous forces $\left(f_{v}\right)$ by Osborne Reynolds about a hundred years ago [16].

$$
R e=\frac{f_{i n}}{f_{v}}
$$

So when the Reynolds number is far below 1, the viscous forces dominate the inertial forces. The Reynolds number can be expressed as a function of the relative object velocity to the fluid $(v)$, the characteristic linear dimension $(L)$, the fluid density $(\rho)$ and the fluid dynamic viscosity $(\mu)$ :

$$
R e=\frac{\rho v L}{\mu}
$$

Swimming performances of microscale swimmers are largely limited due to the low Reynolds dynamics [17]. Let us consider an incompressible flow of Newtonian fluids. The incompressible flow assumes constant viscosity. A simplification of the Navier-Stokes equations is expressed as follows :

$$
-\nabla p+\eta \nabla^{2} \vec{v}=\rho \frac{\partial \vec{v}}{\partial t}+\rho(\vec{v} \cdot \nabla) \vec{v}
$$

where $\eta$ is the constant dynamic viscosity, $p$ is the pressure, $\rho$ is the fluid density and $\vec{v}$ is the flow velocity. As viscous forces dominant at low Reynolds number regimes, the inertia terms are negligible. The NavierStokes equation at low Reynolds numbers regimes becomes as following :

$$
\eta \nabla^{2} \vec{v}=\nabla p
$$

This equation is linear and independent of time [18]. The consequence for locomotion is that the flow is reversible at low Reynolds numbers. That implies the reciprocal motion cannot make any movement. That is why only non reciprocal motion, such as corkscrew type rotating propulsion, is suitable for low Reynolds number regimes.

The fluid motion around the helical swimmer is only characterized by one dimensionless parameter : the Reynolds number. Therefore, the propulsion behaviors of the helical swimmers should be the same if they swim at the same Reynolds numbers. To emulate at an upper-scale the environment of microscale robots swimming at low Reynolds numbers, one can use more viscous liquid. In the following experiments, the viscous liquids used are glycerol solutions with different concentrations. Their viscosities were measured by a falling ball viscometer (Brookfield KF10). The viscosity of glycerol is sensitive to environmental temperature. The measured experimental temperature is about $23{ }^{\circ} \mathrm{C}$. The densities of the liquids were measured by a portable density meter (DMA 35). In this paper, for the rotational propulsion characteristics studies, the 
translational velocities were zero. For comparison of the propulsion performances at each Reynolds number in function of fluid viscosity, the two terms translational velocity and characteristic linear dimension should be kept as constants. In order to calculate the $R e$ for rotational propulsion characteristics, we assumed that the linear velocities were 1 pitch per second. The characteristic linear dimensions were taken as the hydraulic diameters of the SHNs. The hydraulic diameter of a tube is defined as the difference between its outer diameter and inner diameter, which is its thickness. Table II summarizes the measured viscosities of glycerol solutions used and the calculated $R e$ at which the two sizes of SHNs swim in the rotational propulsion experiments.

TABLE II: Measured viscosity of different glycerol solution and calculated Re at which SHN10s and SHN2 swim in the rotational propulsion experiments.

\begin{tabular}{l|llll} 
\% of Glycerol & $\begin{array}{l}\text { viscosity } \\
(\mathrm{mPa} \cdot \mathrm{s})\end{array}$ & $\begin{array}{l}\text { density } \\
\left(\mathrm{g} / \mathrm{m}^{3}\right)\end{array}$ & $\operatorname{Re}(\mathrm{SHN} 10)$ & $\operatorname{Re}(\mathrm{SHN} 2)$ \\
\hline $30 \%$ & 3.2 & 1.09 & - & 0.54 \\
$50 \%$ & 8.5 & 1.14 & 2.68 & - \\
$60 \%$ & 16.1 & 1.17 & 1.45 & 0.11 \\
$70 \%$ & 32.4 & 1.19 & 0.73 & 0.06 \\
$75 \%$ & 43.7 & 1.20 & 0.54 & - \\
$80 \%$ & 66.5 & 1.21 & 0.36 & 0.03 \\
$85 \%$ & 124.6 & 1.22 & 0.20 & - \\
$90 \%$ & 260.0 & 1.23 & 0.09 & 0.008
\end{tabular}

In water, a swimming bacterium such as E.coli has a $R e \approx 10^{-5}-10^{-4}$. A human spermatozoon moves with $R e \approx 0.01$. The larger ciliates such as paramecium swims with $R e \approx 0.1$ [18]. The HNBs swim with $R e \approx$ $3.4 \times 10^{-4}-3.6 \times 10^{-2}$ [10]. In the following experiments for characterization of rotational propulsion properties, the $R e$ at which the SHNs swim were $R e \approx 0.008-3$. It is technically difficult to further decrease the $R e$ at which our SHNs swim. Nevertheless, with these experimental conditions, the rotational propulsion behaviors of the SHNs were studied at low Reynolds numbers.

\section{Experimental setup}

Researchers utilized orthogonal arrangements of electromagnetic coil pairs to generate rotating uniform magnetic fields at the center of the system's workspace to propel the helical swimmers [7], [8]. However, these coil pairs are difficult to be scaled up [17]. Moreover, the energy efficiency largely decreases when we scaled them up. Instead of the coil pairs, a rotating permanent magnet manipulator allows to control a scaled-up models wirelessly [19], [20], [21]. The magnet used in the experiments is cylindrical, $60 \mathrm{~mm}$ in length and $15 \mathrm{~mm}$ in diameter, mounted on a Maxon DC motor. The rotation frequency of the motor is controlled. The chosen configuration of the magnetic actuation is lateral. In this configuration, the SHNs can be more proximate to the magnet. Later, the rotating magnetic field can be combined with a translational movement by a linear motion stage (Newmark systems ET250) to generate a magnetic gradient. The SHNs are placed $25 \mathrm{~mm}$ away from the axis of the magnet. The magnetic field strength around the functional parts (magnetic parts) of the helical swimmers is about $30 \mathrm{mT}$. The magnetic field magnitudes around the permanent magnet manipulator were measured using a Hirst GM08 gaussmeter, and are depicted by Fig $4 b$, in which the magnet is put along the $\mathrm{x}$ axis, and the center of the magnet is at the origin.

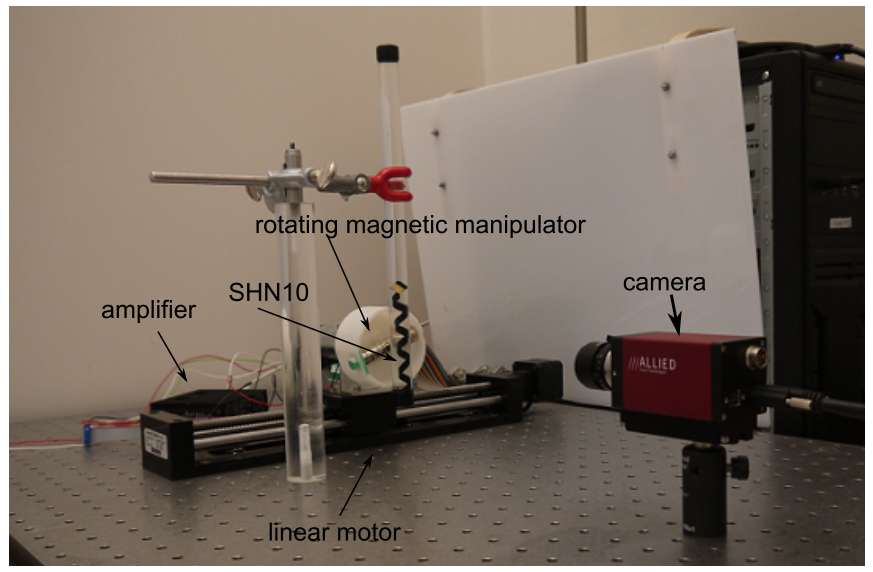

(a)

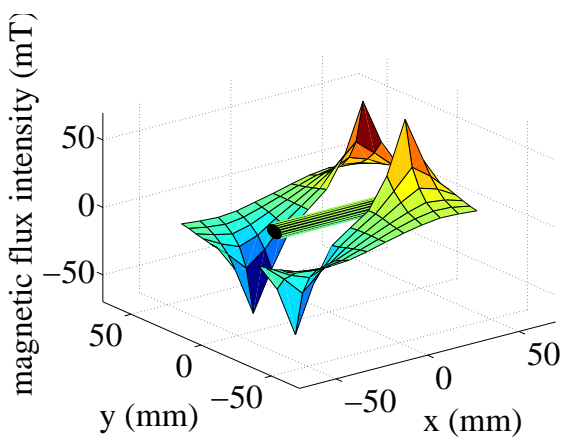

(b)

Fig. 4: (a) Photo of the experimental setup. A SHN10 with a magnetic square head is swimming inside a transparent tube which is placed before a rotating magnet manipulator. (b) Magnetic field magnitudes measured around the magnet. The black cylinder presents the magnet.

The camera used in the experiments was a PIKE F032C firewire camera. In the experiments, the rotations of the SHNs are recorded by the camera. The rotation frequency of the SHN is calculated off-line by the time that a SHN takes to rotate one turn. The frame rate used is about 50 frames per second. The maximal SHN's rotation frequency measured in the experiments is about $20 \mathrm{~Hz}$. The maximal rotation frequency error is estimated at about $2 \%$. The linear velocity is measured 
off-line by the travelled distance in pixel per unit time. Then, it is converted in international unit. The maximal linear velocity error is estimated at about $5 \%$.

\section{MODELLING OF HELICAL SWIMMERS}

\section{A. Helical propulsion}

We consider only two degrees of freedom of a helical swimmer : rotation at angular speed $\omega$ around its axis and translation at velocity $v$ along that axis. These motions are caused by some external force $F$ and some external torque $\tau$ applied to the helical swimmer. At the same time, a force $-F$ and a torque $-\tau$ are exerted on the helical swimmer by the surrounding fluid. According to the equation (4), the $F$ and $\tau$ must be linearly related to the $v$ and $\omega$ [2] :

$$
\begin{aligned}
F & =a v+b \omega \\
\tau & =c v+d \omega
\end{aligned}
$$

The $2 \times 2$ matrix $\left[\begin{array}{ll}a & c \\ b & d\end{array}\right]$ is called the propulsion matrix $P$ of the helical swimmer. The hydrodynamicists call this matrix the resistance matrix. The constants a, $\mathrm{b}, \mathrm{c}$ and $\mathrm{d}$ are proportional to the fluid viscosity $\eta$ and depend otherwise only on the shape and the size of the swimmer. If every dimension of the swimmer is increased by the factor $k$, the new propulsion matrix $P^{\prime}$ has elements $a^{\prime}=k a, b^{\prime}=k^{2} b, c^{\prime}=k^{2} c$ and $d^{\prime}=k^{3} d$ [2]. Purcell proved in his paper [3] that the propulsion matrix must be symmetrical $(b=c)$. The motion is actually described by only three constants. He pointed out that the propulsive efficiency is more or less proportional to the square of the off-diagonal element of the matrix.

In conlusion, at low Reynolds number, the relationship between the external nonfluidic force $(F)$ and nonfluidic torque $(\tau)$ that act on a helical swimmer and its linear and rotational velocities $(v)$ and $(\omega)$ can be written by :

$$
\left[\begin{array}{l}
F \\
\tau
\end{array}\right]=\left[\begin{array}{ll}
a & b \\
b & c
\end{array}\right]\left[\begin{array}{l}
v \\
\omega
\end{array}\right]
$$

where $a, b$ and $c$ are the parameters of the propulsion matrix of the helical swimmer.

\section{B. Nonfluidic forces and torques}

1) Magnetic forces and torques: The helical swimmers are actuated by a rotating magnetic field. All magnetized objects within an externally imposed magnetic field will have both forces and torques exerted on them. Magnetic forces $\left(\overrightarrow{f_{m}}\right)$ are proportional to the gradient of the magnetic field $(\nabla \vec{B})$ in the neighbourhood of the magnetized object, and act to move the object to local maxima. Magnetic torques $\left(\overrightarrow{\tau_{m}}\right)$ are proportional to the magnetic field $(\vec{B})$ and act to align the internal magnetization of an object $(\vec{M})$ with the field. We suppose that the magnetization of the object is uniform. The equations that express the interactions are as follows [22] :

$$
\begin{aligned}
& \overrightarrow{f_{m}}=V_{m}(\vec{M} \cdot \nabla) \vec{B} \\
& \overrightarrow{\tau_{m}}=V_{m} \vec{M} \times \vec{B}
\end{aligned}
$$

where $V_{m}$ is the volume of the magnetized object. An applied magnetic field at the location of the magnetized object transduces into torque and the spatial magnetic gradient transduces into applied force.

2) Apparent weight: The apparent weight is the net force between gravitational force and buoyancy force, which is defined as :

$$
\overrightarrow{f_{w}}=\left(\rho_{(S H N)}-\rho_{f}\right) \vec{g} V_{(S H N)}
$$

where $\rho_{(S H N)}$ is the density of the SHN, $\rho_{f}$ is the density of the fluid and $V_{(S H N)}$ is the volume of the SHN, which is shown in Table I.

3) Friction force: Friction is the force resisting the relative motion of solid surfaces, fluid layers and material elements sliding against each other. Friction force is proportional to the apparent weight :

$$
f_{F}=\mu f_{w}
$$

where $\mu$ is the coefficient of friction.

\section{Modelling of the helical swimmer with square magnetic head}

We first model the helical swimmer with a square magnetic head (SHN10-magSquare). The SHN10magSquare is put before the rotating magnet manipulator, along the $x$ axis. The magnetization (M) direction of the SHN10-magSquare is along the longer edge of the square magnet on its head, as shown in Fig. 6a. The initial position of the magnet manipulator is along the $x$ axis, as shown in Fig. $5 \mathrm{a}, \mathrm{b}$ and c. The magnetic field generated by the rotating permanent magnet manipulator is simulated by Comsol. The red arrows represent the magnetic field. According to Equation (9), the magnetic torque around the $x$ axis is zero (i.e. $\left.(\vec{M} \times \vec{B}) \cdot \overrightarrow{u_{x}}=0\right)$ at the initial position. After the magnet manipulator turns $45^{\circ}$, the magnetic field near the head position of the SHN10-magSquare turns at an angle around the $x$ axis, as shown in Fig. $5 \mathrm{~d}$. If the SHN10-magSquare stays in its initial position, it will be subject to a magnetic torque around the $x$ axis (i.e. $\left.(\vec{M} \times \vec{B}) \cdot \overrightarrow{u_{x}} \neq 0\right)$. Thus, the SHN10-magSquare will follow the magnetic field. The magnetic field near the head position of the SHN10-magSquare projected on the plane yoz rotates a whole turn around the $x$ axis, if the magnet manipulator rotates one turn. As a result, the SHN10-magSquare rotates around the $x$ axis by the action of the rotating magnetic field. 


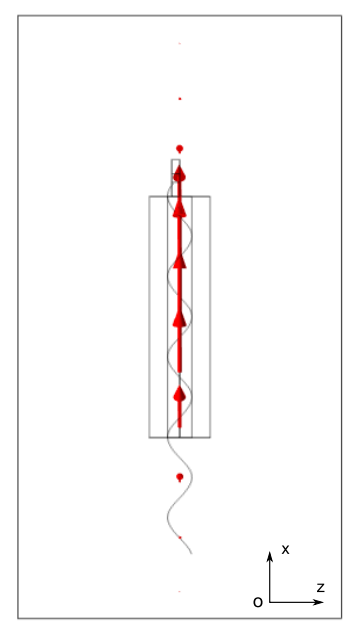

(a)

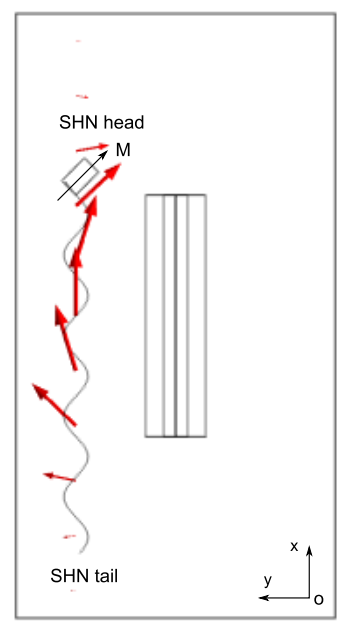

(b)

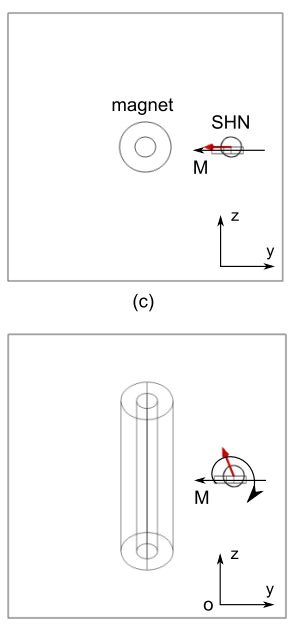

(d)

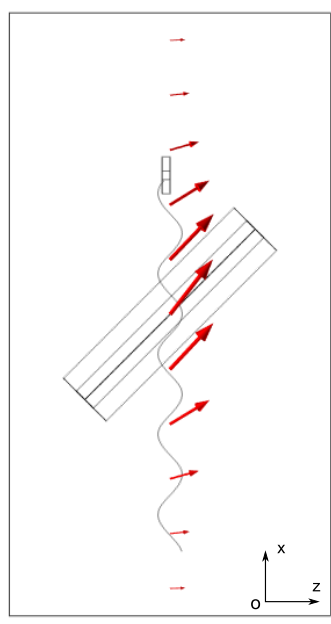

(e)

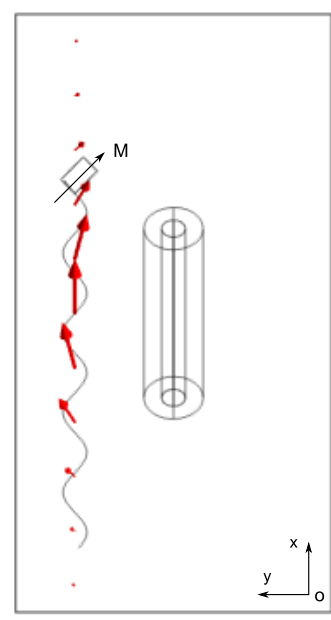

(f)

Fig. 5: (a), (b) and (c) The red arrows represent respectively the magnetic field generated by rotating magnet manipulator around the magnetic head at initial position $\left(0^{\circ}\right.$ configuration $)$ in the plane xoz, xoy and yoz. The black arrows represent the magnetization of magnetic head. (e), (f), and (d) represent respectively (a), (b), and (c) with the rotating magnet in a $45^{\circ}$ configuration.

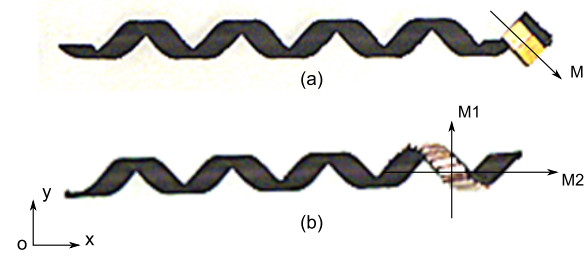

Fig. 6: (a) The magnetization (M) direction of the SHN10-magSquare is along the longer edge of the square magnet on its head. (b) The helical swimmer with a magnetic tail (e.g. SHN10-none) has two directions of magnetization. M1, which is perpendicular to the $x$ axis, contributes to propulsion.

\section{Modelling of the helical swimmer with magnetic tail}

The swimmers with helical magnetic tail have two directions of magnetization. One is perpendicular to their axis (M1), the other one is along their axis (M2), as shown in Fig. 6b. M1 contributes to propulsion, because the magnetic field rotates around the $x$ axis which is the axis of the helical swimmer. A volumetric magnetic torque exerts on the helical swimmer around the $x$ axis, according to Equation (9). A simulation of the helical swimmer exerted a volumetric torque around its axis with one end fixed shows how the helical swimmer deforms. This deformation of the helical swimmer is shown in Fig. 7 The red arrows represent the displacements of the points at the end of the arrows. It shows that the helical swimmer is twisted. If, instead of being fixed, its end was free, the helical swimmer would then rotate. The rotation direction of a magnetic actuated helical swimmer can be easily changed by reversing the rotation direction of the magnetic field.

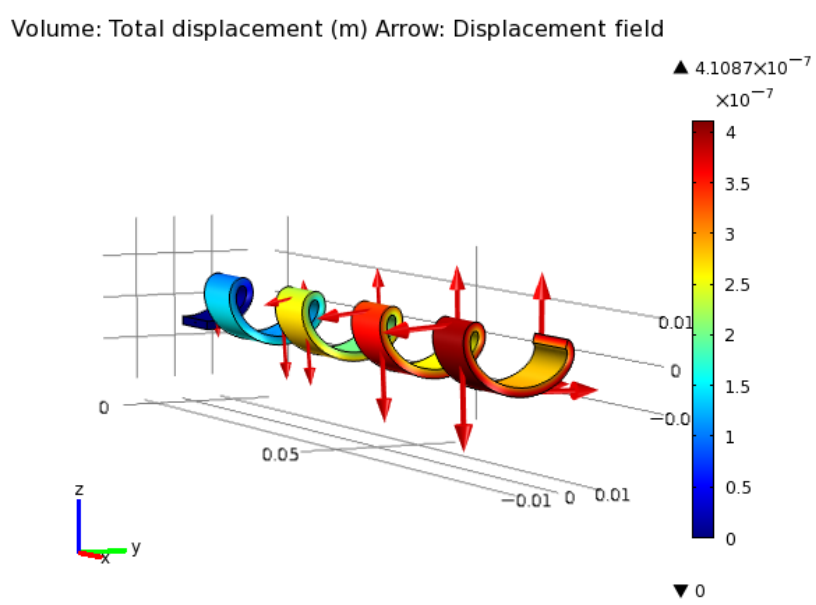

Fig. 7: The deformation simulation of the SHN10-none exerted a volumetric torque around its axis with one end fixed.

\section{EXTERNAL FORCE AND TORQUE ANALYSES BASED ON PROPULSION MATRICES}

\section{A. Propulsion matrix identification}

The $a, b, c$ parameters in (7) need to be identified for the estimation of the force and torque necessary to drive a helical swimmer at certain angular and linear speeds. Two experiments are necessary for the identification of the propulsion matrix. The first experiment consists in dropping the helical swimmer freely by placing the tube vertically, and the second one consists in dropping down helical swimmer within an active rotating magnetic field. The sign convention is defined as that the downward direction is positive for $f$ and $v$. 
The right-handed chirality rotation direction is defined as positive for $\omega$ and $\tau$.

In this section, the propulsion matrices of SHN10none and SHN10-full are estimated in different viscous liquid. In the first experiment, the external nonfluidic torque is null. The external nonfluidic applied force is the apparent weight of the helical swimmer, which results from its gravity force and buoyancy force. This nonfluidic force is estimated as $0.01 \mathrm{~N}$ for SHN10-none, and $0.035 \mathrm{~N}$ for SHN10-full. In the second experiment, the rotating magnetic field exerts a magnetic torque on the helical swimmer. The helical swimmer rotates under the rotating field. The rotation of the helical swimmer generates an upward propulsive force which slows down the dropping speed. If this propulsive force is strong enough to balance the apparent weight, the helical swimmer can be held in the vertical position. Yet, in our case, this balance is not reached because of the cut-off frequency of the helical swimmer which is explained in section. V-A.

This magnetic force exerted on the helical swimmer is independent on the rotation frequency. First, SHN10none dropped within rotating magnetic fields with different rotation frequency values were measured in $75 \%$ glycerol. The vertical linear velocity and angular speed are noted as $v_{i}$ and $\omega_{i}$ for rotating magnetic field of $i \mathrm{hz}$. We can identify the propulsion matrix from the measures of dropping for 3 rotating frequency values, yielding 3 quadruplets $\left(f_{i}, \tau_{i}, v_{i}, \omega_{i}\right), i=0 . .2$, where only $v_{i} \mathrm{~s}$ and $\omega_{i} \mathrm{~s}$ can be measured. Indeed, from (7), we have :

$$
f_{i}=a v_{i}+b \omega_{i}, i=0 . .2
$$

Under the assumption that the magnetic forces of different rotating fields are the same, this can be used to write :

$$
f_{1}=a v_{1}+b \omega_{1}=f_{2}=a v_{2}+b \omega_{2}
$$

This yield :

$$
a\left(v_{1}-v_{2}\right)+b\left(\omega_{1}-\omega_{2}\right)=0
$$

For the specific case where the rotating frequency is $0 \mathrm{~Hz}$, we know that $f_{0}$ is the measured apparent weight. Thus:

$$
f_{0}=a v_{0}+b \omega_{0}
$$

The parameters $(a, b)$ are thus the solution to the linear system formed by the last two equations (14) and (15).

$$
\begin{aligned}
a & =\frac{f_{0}}{v_{0}-\frac{\omega_{0}}{\omega_{1}-\omega_{2}}\left(v_{1}-v_{2}\right)} \\
b & =-\frac{v_{1}-v_{2}}{\omega_{1}-\omega_{2}}
\end{aligned}
$$

From (7), we also have :

$$
\tau_{0}=b v_{0}+c \omega_{0}
$$

TABLE IV: Summary of $a, b, c$ parameters of propulsion matrices for SHN10-none and SHN10-full in different viscous liquid.

\begin{tabular}{l|llll} 
Type & $\begin{array}{l}\% \text { gly- } \\
\text { cerol }\end{array}$ & $\begin{array}{l}\text { a } \\
(\mathrm{N} \cdot \mathrm{s} / \mathrm{m})\end{array}$ & $\mathrm{b}(\mathrm{N} \cdot \mathrm{s})$ & $\begin{array}{l}\mathrm{c} \\
(\mathrm{N} \cdot \mathrm{m} \cdot \mathrm{s})\end{array}$ \\
\hline \multirow{2}{*}{ SHN10-none } & 50 & 0.06 & $-1.3 \times 10^{-4}$ & $1.8 \times 10^{-6}$ \\
& 75 & 0.11 & $-1.5 \times 10^{-4}$ & $2.7 \times 10^{-6}$ \\
\hline \multirow{2}{*}{ SHN10-full } & 50 & 0.09 & $-1.2 \times 10^{-4}$ & $1.9 \times 10^{-6}$ \\
& 75 & 0.19 & $-2.9 \times 10^{-4}$ & $3.3 \times 10^{-6}$
\end{tabular}

where $\tau_{0}$ is the applied external magnetic torque for free dropping of the helical swimmer. There is no external magnetic field, thus the applied magnetic torque is zero. We can get :

$$
c=\left(\tau_{0}-b v_{0}\right) / \omega_{0}
$$

The numerical values of $a, b, c$ parameters obtained in the experimental conditions of Table III are given in Table IV.

The parameter $a$ is expressed in $\mathrm{N} \cdot \mathrm{s} / \mathrm{m}, b$ is expressed in $\mathrm{N} \cdot \mathrm{s}$ and $c$ is expressed in $\mathrm{N} \cdot \mathrm{m} \cdot \mathrm{s}$.

The external nonfluidic force and torque of SHN10none are now calculated based on the estimated propulsion matrix. The external nonfluidic force results from the apparent weight and the magnetic force. From the nonfluidic force calculated in Table III, we get that the magnetic force is negligeable. This is validated by some more measures of the dropping within $3 \mathrm{~Hz}$ and $4 \mathrm{~Hz}$ rotating magnetic field. Only two measures are necessary to determine the $a, b, c$ parameters of the propulsion matrix of a helical swimmer.

The parameters of the propulsion matrices of SHN10none and SHN10-full in 50\% and $75 \%$ glycerol are summarized in Table IV. The Reynolds number calculated with dropping velocities are $R e=13.4-26.8$ for SHN10-none in 50\% glycerol, $R e=1.37-2.75$ in $75 \%$ glycerol, and $R e=40.2-53.8$ for SHN10-full in 50\% glycerol, $R e=2.76-5.49$ in $75 \%$ glycerol. The Reynolds numbers are relatively high for SHNs in $50 \%$ glycerol, because the dropping velocities are important. All the $a$ and $c$ parameters are positive and all the $b$ parameters are negative. The absolute values of $a, b, c$ decrease with the Reynolds number. The absolute values of $a, b, c$ of SHN10-full are generally higher than that of SHN10none.

\section{B. Force and torque analyses}

This section contributes to the force and torque analyses based on the propulsion matrix estimated in section IV-A. The helical swimmer was driven by the combination of rotating magnetic field and magnetic gradient. The torque exerted on the helical swimmer is the magnetic torque generated by the rotating magnetic field. The force results from the magnetic force generated by the magnetic gradient and the propulsive force generated by its rotation. The input parameters of the 
TABLE III: Measurements of dropping velocities and angular speeds of SHN10-none and SHN10-full in different viscous liquid in function of the rotation frequency of the applied magnetic field, and the nonfluidic forces and torques calculated from estimated propulsion matrices.

\begin{tabular}{l|ccccc|cc|cccc} 
Type & \multicolumn{1}{|c|}{ SHN10-none } & \multicolumn{3}{c}{ SHN10-full } \\
\hline Reynolds number & \multicolumn{4}{c|}{$75 \%$ glycerol } & $50 \%$ glycerol & $50 \%$ glycerol & $75 \%$ glycerol \\
\hline Rotation frequency (hz) & 0 & 1 & 2 & 3 & 4 & 0 & 3 & 0 & 3 & 0 & 1 \\
Vertical velocity (mm/s) & 102 & 83 & 74 & 66 & 52 & 213 & 147 & 432 & 370 & 208 & 171 \\
Angular speed (rad/s) & 5.7 & -6.3 & -12.6 & -18.8 & 14.8 & -14.4 & -25.1 & 27.3 & -19 & 18.4 & -6.3 \\
Nonfluidic force $\left(10^{-2} \mathrm{~N}\right)$ & 1.02 & 1.02 & 1.04 & 1.06 & 1.02 & 1.09 & 1.07 & 3.56 & 3.56 & 3.44 & 3.45 \\
Nonfluidic torque $\left(10^{-5}\right.$ N.m) & $\approx 0$ & -3.44 & -5.24 & -7.04 & -8.72 & $\approx 0$ & -4.50 & $\approx 0$ & -8.05 & $\approx 0$ & -7.06
\end{tabular}

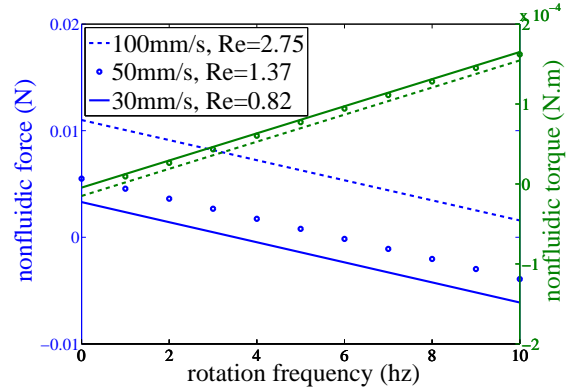

(a)

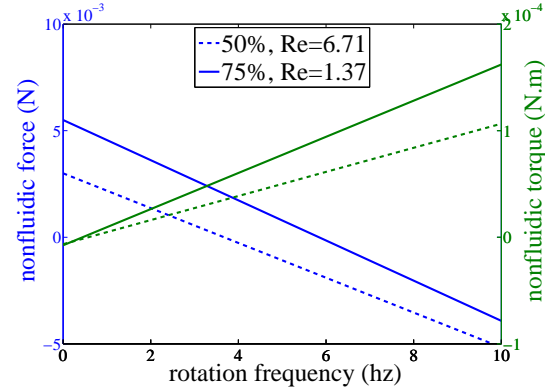

(b)

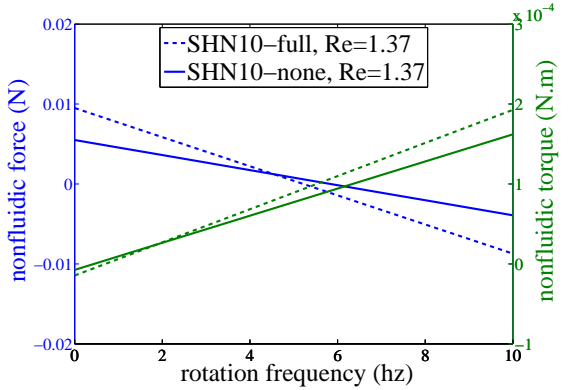

(c)

Fig. 8: (a) Required external force and torque for SHN10-none in function of rotation frequency in $75 \%$ glycerol. (b) Required force and torque for translating SHN10-none at $50 \mathrm{~mm} / \mathrm{s}$ at different Re. (c) Required force and torque for SHN10-none and SHN10-full in function of rotation frequency for a desired translation velocity at $50 \mathrm{~mm} / \mathrm{s}$.

system are the rotation frequency of the rotating magnetic field and the velocity of the linear motion stage. The outputs of the system are the rotation frequency and translation velocity of the helical swimmer.

Fig. 8a shows the required force and torque for SHN10-none in function of the rotation frequency for different desired translation velocities based on the propulsion matrix calculated for $75 \%$ glycerol. The Reynolds numbers calculated with these translation velocities are $R e=0.82-2.75$. More external force was required for SHN10-none to translate with higher velocity but the same rotation frequency. The required external force decreased with the rotation frequency, because the more propulsive force was generated by higher rotation frequency. In the balance case, the propulsive force can keep the helical swimmer advancing, and no external force should be required. For example, in ideal conditions, SHN10-none rotating at $1.5 \mathrm{~Hz}$ can provide a propulsive force for a translation at $30 \mathrm{~mm} / \mathrm{s}$, because the pitch of SHN10-none is $20 \mathrm{~mm}$. Yet in the experimental conditions, SHN10-none had to rotate at about $2 \mathrm{~Hz}$ to keep a $30 \mathrm{~mm} / \mathrm{s}$ translation. This additional propulsive force was to counterbalance the friction with the tube wall.

Naturally, the translation of a helical swimmer creates a rotation in a viscous liquid. It explains why a negative torque is required to keep SHN10-none translating without rotation. The required external torque increased with the rotation frequency. As translational motion creates a rotation of the helical swimmer, less external torque is required for a SHN10-none with higher translation velocity but the same rotation frequency.

Fig. $8 \mathrm{~b}$ describes the required torque and force to keep SHN10-none translating at $50 \mathrm{~mm} / \mathrm{s}$ at different Reynolds numbers. The calculated Reynolds numbers are $R e=1.37$ for $75 \%$ glycerol, and $R e=6.71$ for $50 \%$ glycerol. More external torque and force is required if SHN10-none swims at lower Reynolds number. SHN10-none rotates faster in less viscous liquid with the same translation velocity and without additional external torque.

Fig. $8 \mathrm{c}$ describes the required force and torque for SHN10-none and SHN10-full in function of the rotation frequency in $75 \%$ glycerol for a $50 \mathrm{~mm} / \mathrm{s}$ translation velocity. The calculated Reynolds number is $R e=1.37$. We need more torque in absolute value for SHN10full than for SHN10-none, because SHN10-full has a higher inertia. The decrease rate of external force required for SHN10-full is higher than for SHN10-none. It implies the propulsive force created by SHN10-full is greater than that of SHN10-none at the same rotation frequency. 


\section{CHARACTERISTICS OF SCALED-UP HELICAL MICROSWIMMERS AND THE INFLUENCE FACTORS}

\section{A. Propulsion characteristics of SHN10s without a head}

In this section, the two helical swimmers without heads, SHN10-none and SHN10-full were tested at different Reynolds numbers. Within a rotating magnetic field generated by the rotating permanent magnet manipulator, the SHN10-none rotated at first synchronously with the applied field until a maximal rotation frequency. Beyond this maximal rotation frequency, it suddenly stopped rotating, but started to oscillate. We call this maximal rotation frequency as a cut-off frequency. Fig. 9a and $9 \mathrm{~b}$ show respectively the rotation frequency of the SHN10-none and SHN10-full in function of the rotation frequency of the magnetic field at different Reynolds numbers.

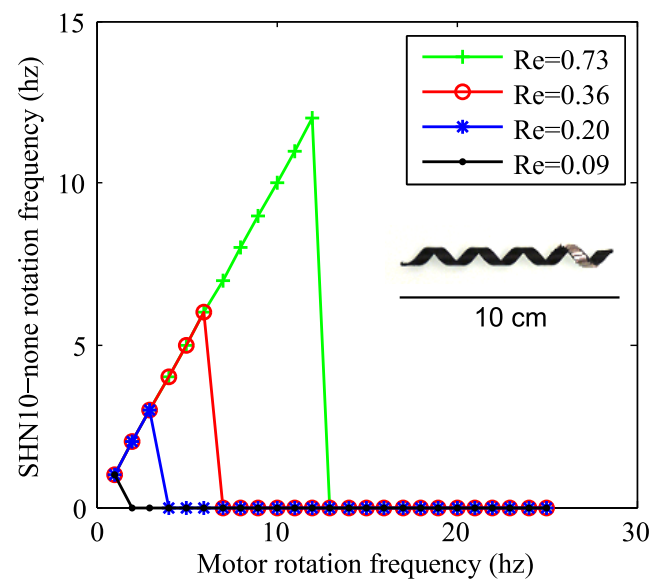

(a)

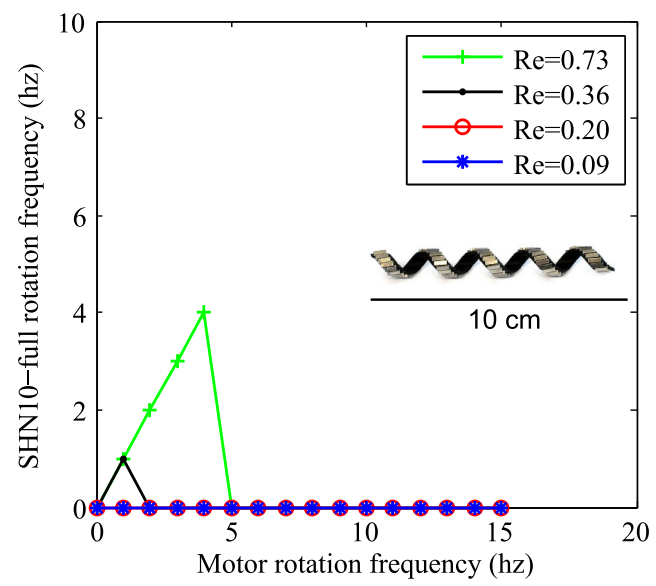

(b)

Fig. 9: The rotation frequency of (a) SHN10-none and (b) SHN10-full in function of the rotating magnetic field's frequency at different Reynolds numbers.

These cut-off frequency values strongly depend on the Reynolds number at which the helical swimmers swim, because they have to overcome the rotational fluidic drag torque which depends on the viscosity of the liquid. The more viscous the liquid is, the stronger the fluidic drag torque is. Thus, the cut-off frequency decreased with the Reynolds number. Beyond this cutoff frequency, the available magnetic torque was no longer sufficient to keep the helical swimmers rotating in sync with the applied field.

The cut-off frequency of the SHN10-full was much smaller than that of the SHN10-none for the same frequency of the rotating field at the same Reynolds number. The inertia of SHN10-full is higher than that of SHN10-none. Therefore, more torque was required to rotate SHN10-full than SHN10-none with the same rotation frequency. The helical swimmer with its first pitch magnetic showed better rotational propulsion performance than the helical swimmer with its entire surface magnetic. In the following experiments, the helical swimmers with just one pitch magnetic were used.

\section{B. Head influence on swimming characteristics}

In this section, we designed a series of experiments to show the influence of head shapes on propulsion characteristics of helical swimmers. The SHN10-magTails (SHN10s with their first pitches magnetic) with three different head shapes : SHN10-cylinder, SHN10-sphere and SHN10-square, were tested in the following experiments.

Fig. 10a shows the rotation frequency of SHN10cylinder in function of the rotating field frequency at different Reynolds numbers. SHN10-cylinder showed as well a cut-off frequency with a brutal stop. Its rotational propulsion characteristics curve shapes are similar than that of SHN10-none as shown in Fig. 9a. The rotational propulsion characteristics curve shapes of SHN10-square and SHN10-sphere are also similar : synchronization with the rotating field and brutal stop. Fig. 11 summarizes the cut-off frequency values of the helical swimmers with magnetic tail and with different heads in function of $R e$. The SHN10-none have the highest cut-off frequency values, because the SHN10none do not have a head that creates additional fluidic drag torque. The square head created more drag torque than the spherical and cylindrical heads.

In conclusion, the head shape of a helical swimmer does not influence the shape of the rotational propulsion characteristics curve, but it influences the cut-off frequency values. As these values do not differ much, this influence is not significant.

\section{Magnetic positioning influence on swimming characte-} ristics

The following experiments were designed in order to compare the rotational propulsion characteristics between a helical swimmer with a magnetic tail and a helical swimmer with a magnetic head. Only a helical swimmer with a square plate head (SHN10- 


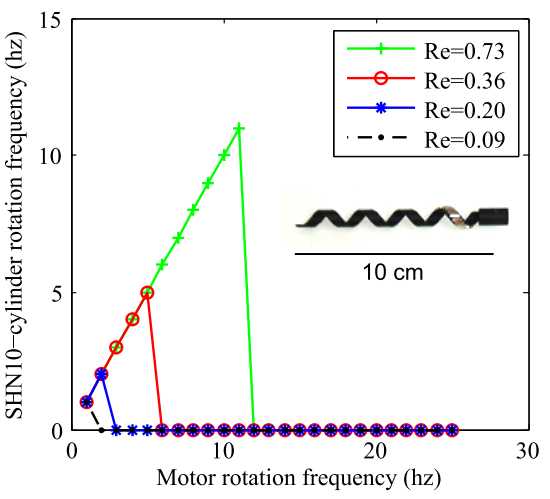

(a)

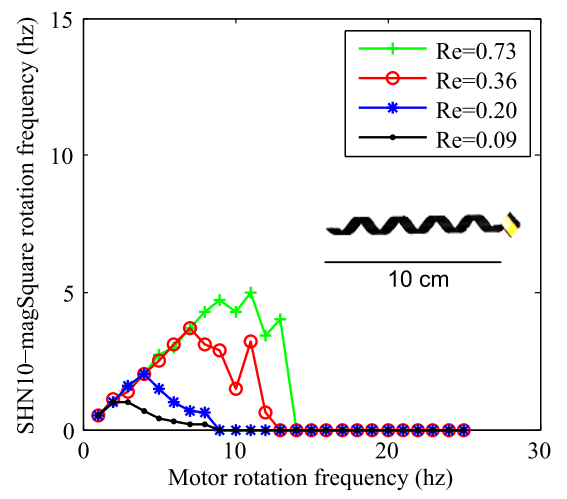

(b)

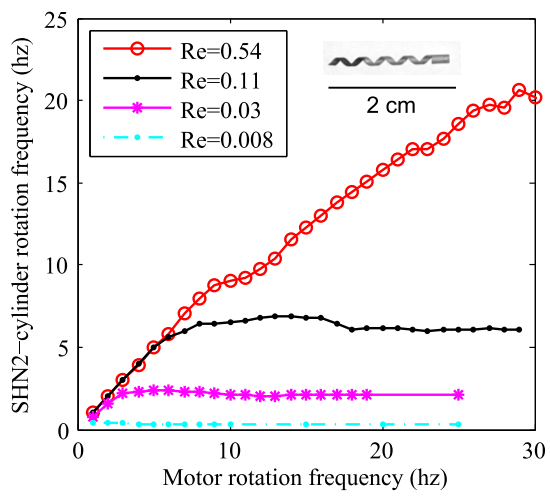

(c)

Fig. 10: (a) The SHN10-cylinder shows a cut-off frequency with a brutal stop. (b) The SHN-magSquare shows a cut-off frequency with a gentle decline. (c) The SHN2-cylinder shows a saturation of frequency.

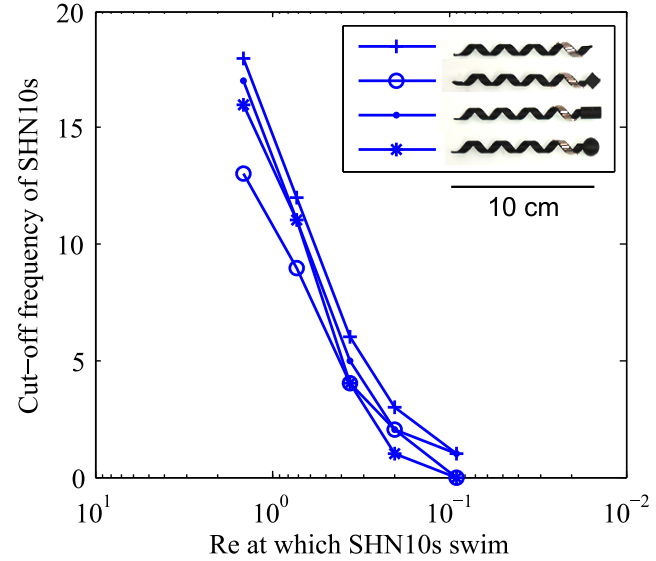

Fig. 11: The maximal propulsive speed of helical swimmers with different heads but the same magnetic tail in function of Reynolds numbers.

magSquare) was used, because it was not easy to cover other heads with magnets.

The SHN10-magSquare showed different rotational propulsion characteristics from the SHN10s with magnetic tails. Fig. 10b presents the rotational propulsion characteristics of SHN10-magSquare at different Reynolds numbers. The rotation frequency of the SHN10magSquare was about half of the frequency of the rotating magnetic field until it reached the maximum. It is caused by the head being attracted by the magnet manipulator, a strong resistant torque was created when the two side corners of the head hit on the tube wall. The magnetic torque was not strong enough to overcome this resistant torque. Thus the rotation frequency of the SHN10-magSquare was divided by two. If the attraction acted on the tail of the SHN10-Square, its head would not hit the tube wall. Only a weak torque was created by the friction between the lateral surface of its tail and the tube wall. The SHN10-Square thus rotated synchronously with the rotating field.
After the maximum, the rotation frequency of SHN10magSquare decreased if the rotation frequency of the magnetic field increased further more. The SHN10magSquare showed a cut-off frequency with a gentle decline. After the cut-off frequency, the decrease of the rotation frequency was not regular. At $R e=0.09$ and $R e=0.20$, the decrease was monotone and almost linear. At $R e=0.36$ and $R e=0.73$, the decrease was not stable. Some rebounds existed, but the overall trend was that the rotation frequency decreased towards zero.

The helical swimmers with magnetic heads have different rotational propulsion characteristics than those of the helical swimmers with magnetic tails. We discuss here the reason which causes this difference. The magnetization direction of the SHN10-none with magnetic tail, which contributes to propelling itself by following the rotating magnetic field, is perpendicular to its axis (M1 in Fig. 6). We defined each possible magnetization direction of the helical swimmer which contributes to propulsion as a magnetization phase. The SHN10-none has several magnetization phases, because its first pitch is covered by 17 magnets. On the opposite, the SHN10-magSquare with a magnetic head has just one magnetization phase, which is in the plane of the magnetic square plate. We presume that this number of magnetization phases influences the rotational propulsion characteristics of the helical swimmer.

\section{Magnetization phase influence on swimming characte- ristics}

In order to validate the presumption that propulsion characteristics are influenced by the number of magnetization phases, the following experiments were designed.

The SHN2-cylinder with its helical tail uniformly coated by magnetic materials has much more magnetization phases than the SHN10-none, because the later has only 17 magnets covered on its first pitch. Since 
the SHN2-cylinder is uniformly coated, its number of magnetization phases is considered as near infinity. The propulsion characteristics of the SHN2-cylinder at different Reynolds numbers is shown in Fig. 10c. The rotational propulsion characteristics curve of SHN2cylinder is different from those of the SHN10s with magnetic tails and SHN10-magSquare. The rotation frequency of the SHN2-cylinder rotated in sync with the rotating magnetic field at the beginning, then the increase rate decreased, and finally the rotation frequency stabilized at a value which was slightly smaller than its maximum. This value is called as the saturation frequency of the SHN2-cylinder. The increase step and the saturation frequency of the SHN2-cylinder depend strongly on the Reynolds number. At relatively high $R e(R e=0.54)$, the rotation frequency of the SHN2cylinder increased continuously, and the saturation frequency was not reached before $25 \mathrm{~Hz}$. At relatively low $R e(R e=0.008)$, the saturation frequency was reached before $1 \mathrm{~Hz}$. The increase step was not shown.

It means that the number of magnetization phases is an influencing factor of the rotational propulsion characteristics of helical swimmers . The rotational propulsion characteristics of the three different helical swimmers with different magnetization phases are different. The SHN10-magSquare with 1 magnetization phase showed a cut-off frequency with a gentle decline towards zero. The rotation frequency of the SHN10magSquare was about half of the frequency of the rotating field. The SHN10s with magnetic tails with several magnetization phases rotated in sync with the rotating field. However, after it lost the synchronization with the rotating field, it came to a brutal stop. The SHN2cylinder with a uniformly coated magnetic tail has near infinity magnetization phases, which showed a saturation of frequency. The rotation frequency increased synchronously with the rotating field, then the increase rate decreased and the rotation frequency stabilized at a value slightly smaller than its maximal rotation frequency. This stable value of rotation frequency is called the saturation frequency.

\section{CONCLUSION}

In this paper, we proposed a scaled-up experimental system for preliminary analyses of helical swimmers swimming abilities. This scaled-up system consists in a rotating permanent magnet driving scaled-up helical microswimmers. We proved in this paper that a helical swimmer with a magnetic tail and without a head can rotate within a rotating magnetic field. Therefore, a magnetic head is not necessary to drive a helical swimmer.

The parameters of the propulsion matrices of SHN10none and SHN10-full in different viscous liquids were identified. The method of propulsion matrix identification is able to be scaled-down. The required external
TABLE V: Summary of the different types of the scaledup helical swimmers.

\begin{tabular}{l|lll} 
Model & $\begin{array}{l}\text { Magnetic } \\
\text { part }\end{array}$ & $\begin{array}{l}\text { Number of } \\
\text { magnetization } \\
\text { phases }\end{array}$ & $\begin{array}{l}\text { Swimming } \\
\text { characteristics }\end{array}$ \\
\hline SHN10-none & tail & several & cut-off brutal \\
SHN10-cylinder & tail & several & cut-off brutal \\
SHN10-sphere & tail & several & cut-off brutal \\
SHN10-square & tail & several & cut-off brutal \\
SHN10-full & tail & several & cut-off brutal \\
SHN10-magSquare & head & 1 & cut-off gentle \\
tail & infinite & saturation
\end{tabular}

force and torque to drive a helical swimmer were analysed in this paper.

The helical swimmers with their tails covered by small magnets showed cut-off frequency with brutal stop, whatever the head shapes they had : without a head or with cylindrical, spherical, square-plate heads. Below the cut-off frequency, they rotated in sync with the rotating magnetic field. Once the cut-off frequency was reached, the helical swimmers suddenly stop rotating. The cut-off frequency increased with the Reynolds numbers. The helical swimmer without a head had the highest cut-off frequency values, because it did not have a head to create additional fluidic drag torque. The square head created more drag torque than the spherical and cylindrical heads. The head shape does not influence the rotational propulsion characteristics curve, but it influences the cut-off frequency values.

A helical swimmer with a magnetic square head and a non magnetic tail showed a cut-off frequency with gentle decline. Its rotation frequency was about half of the frequency of the rotating field until it reached its maximum, and then its rotation frequency decreased to zero. A helical swimmer with its helical tail uniformly coated by ferromagnetic material showed a saturation of frequency. Its rotation frequency increased synchronously with the rotating magnetic field, then it stabilized at a value slightly smaller than its maximum. The values of cut-off frequency or saturation frequency depend strongly on the Reynolds number. The three types of swimming characteristics are quite different, which is caused by the different number the magnetization phases of helical swimmers. The magnetization phase is defined as a possible magnetization direction of the helical swimmer which contributes to propelling itself. Table $\mathrm{V}$ summarizes the number of magnetization phases and the swimming characteristics of different helical swimmers appeared in the paper.

It is difficult to predict the cut-off frequency values for the helical microswimmers from the results of the scaled-up SHNs, because those values also depend on the strength of the external magnetic field. However, we expect that a microswimmer with a uniformly coated helical magnetic tail will show a saturation of frequency. In this case, the swimming performances will 
not substantially decrease after the cut-off frequency. The characterization of the propulsion behaviors of the microswimmers with uniformly coated ferromagnetic helical tails will be validated in future works.

\section{ACKNOWLEDGMENT}

We acknowledge funding from Émergence-UPMC2012 research program. The authors are also grateful to Sinan Haliyo, Sylvain Pledel, Christophe Grand, Thomas Seon, Tianyi Li and Jean ochin Abrahamians for providing technical supports on the expermental setup.

\section{REFERENCES}

[1] B.J. Nelson, I.K. Kaliakatsos, and J.J. Abbott. Microrobots for minimally invasive medicine. Annual Review of Biomedical Engineering, 12(1) :55-85, 2010.

[2] E.M. Purcell. Life at low reynolds number. American Journal of Physics, 45(1) :3-11, 1977.

[3] E.M. Purcell. The efficiency of propulsion by a rotating flagellum. Proc Natl Acad Sci U S A, 94(21) :11307-11, 1997.

[4] H.C. Berg and R.A. Anderson. Bacteria swim by rotating their flagellar filaments. Nature, 245(5425) :380-382, 1973.

[5] T. Honda, K.I. Arai, and K. Ishiyama. Micro swimming mechanisms propelled by external magnetic fields. Magnetics, IEEE Transactions on, 32(5) :5085 -5087, sep 1996.

[6] L. Zhang, J.J. Abbott, L. Dong, B.E. Kratochvil, D. Bell, and B.J. Nelson. Artificial bacterial flagella : Fabrication and magnetic control. Appl. Phys. Lett., 94(6), 2009.

[7] L. Zhang, K.E. Peyer, and B.J. Nelson. Artificial bacterial flagella for micromanipulation. Lab Chip, 10 :2203-2215, 2010.

[8] Ambarish Ghosh and Peer Fischer. Controlled propulsion of artificial magnetic nanostructured propellers. Nano Letters, 9(6) :2243-2245, 2009.

[9] G. Hwang, S. Haliyo, and S. Régnier. Remotely powered propulsion of helical nanobelts. In Robotics Science and Systems, 2010.

[10] G. Hwang, R. Braive, L. Couraud, A. Cavanna, O. Abdelkarim, I. Robert-Philip, A. Beveratos, I. Sagnes, S. Haliyo, and S. Régnier. Electro-osmotic propulsion of helical nanobelt swimmers. The International Journal of Robotics Research, 30(7) :806-819, 2011.

[11] S. Tottori, L. Zhang, F. Qiu, K. Krawczyk, A. Franco-Obregn, and B.J. Nelson. Magnetic helical micromachines : Fabrication, controlled swimming, and cargo transport. Advanced Materials, 24(6) :pp. 811-816, February 2012. highlighted as the front cover.

[12] K. Ishiyama, K.I. Arai, M. Sendoh, and A. Yamazaki. Spiraltype micro-machine for medical applications. In Micromechatronics and Human Science, 2000. MHS 2000. Proceedings of 2000 International Symposium on, pages $65-69,2000$.

[13] D.J. Bell, S. Leutenegger, K.M. Hammar, L.X. Dong, and B.J. Nelson. Flagella-like propulsion for microrobots using a nanocoil and a rotating electromagnetic field. In Robotics and Automation, 2007 IEEE International Conference on, pages 1128 -1133, april 2007.

[14] L. Zhang, J.J. Abbott, L. Dong, K.E. Peyer, B.E. Kratochvil, H. Zhang, C. Bergeles, and B. J. Nelson. Characterizing the swimming properties of artificial bacterial flagella. Nano Letters, 9 :3663-3667, October 2009.

[15] T. Xu, G. Hwang, N. Andreff, and S. Régnier. Scaled-up helical nanobelt modeling and simulation at low reynolds numbers. In ICRA'12 IEEE International Conference on Robotics and Automation, pages $4045-4051,2012$.

[16] O. Reynolds. An experimental investigation of the circumstances which determine whether the motion of water shall be direct or sinuous, and of the law of resistance in parallel channels. Philosophical Transactions of the Royal Society of London, 174 :pp. 935-982, 1883.
[17] J.J. Abbott, M. Cosentino Lagomarsino, L. Zhang, L. Dong, and B.J. Nelson. How should microrobots swim? The International Journal of Robotics Research, 28(11-12) :1434-1447, 2009.

[18] E. Lauga and T.R. Powers. The hydrodynamics of swimming microorganisms. Reports on Progress in Physics, 72(9) :096601, 2009.

[19] T.W.R. Fountain, P.V. Kailat, and J.J. Abbott. Wireless control of magnetic helical microrobots using a rotating-permanentmagnet manipulator. In Robotics and Automation (ICRA), 2010 IEEE International Conference, pages 576 -581, may 2010.

[20] A.W. Mahoney, D.L. Cowan, K.M. Miller, and J.J. Abbott. Control of untethered magnetically actuated tools using a rotating permanent magnet in any position. In ICRA, pages 33753380. IEEE, 2012.

[21] A.W. Mahoney and J.J. Abbott. Control of untethered magnetically actuated tools with localization uncertainty using a rotating permanent magnet. In Biomedical Robotics and Biomechatronics (BioRob), 2012 4th IEEE RAS EMBS International Conference on, pages $1632-1637$, june 2012.

[22] D. Jiles. Introduction to magnetism and magnetic materials; 2 nd ed. Chapman and Hall, Boca Raton, FL, 1998. 